\title{
Mathematical Modelling of the Screening of Musical Abilities and Skills
}

\author{
Perebinos Mihail ${ }^{1, *}$, Pasat Natalia ${ }^{2}$ \\ ${ }^{1}$ Department of Computer Science and Systems Engineering, Faculty of Computers, Informatics and Microelectronics, Technical \\ University of Moldova (TUM), Chisinau city, Republic of Moldova. \\ ${ }^{2}$ Software and Applications Development and Analysis, Information Technologies in Modeling, Faculty of Physics and Engineering, \\ Moldova State University (MSU), Chisinau city, Republic of Moldova.
}

\begin{abstract}
How to cite this paper: Perebinos Mihail, Pasat Natalia. (2021) Mathematical Modelling of the Screening of Musical Abilities and Skills. Journal of Applied Mathematics and Computation, 5(3), 200-206.

DOI: $10.26855 /$ jamc.2021.09.006
\end{abstract}

Received: August 11, 2021

Accepted: September 3, 2021

Published: September 13, 2021

*Corresponding author: Perebinos Mihail, Department of Computer Science and Systems Engineering, Faculty of Computers, Informatics and Microelectronics, Technical University of Moldova (TUM), Chisinau city, Republic of Moldova.

Email: mihaip52@yahoo.com

\begin{abstract}
Since ancient times, learning and science developed in parallel resulting from the continuous interplay "questions-answers". In this context, due to this approach, the process in Pasat Natalia was focused on research of musical abilities side effects and their degree of influence on individual's multidimensional development. In order to reach an efficient decision-making process, experts were involved to solve an optimization problem linked to reclassifying or supplementing the characteristics set. For this particular case, three criteria were proposed to be considered, each consisting of individual classes of characteristics. Based on Pareto analysis, metrics for each of the local and integrated criteria are presented. Starting with various assumptions and advancing to the obtained results, 4 challenges and 2 remarks, a task was formulated. Using the Churchman-Ackoff method, a "primary" model for quantitative assessment of musical abilities and skills screening, which have a vast impact on overall human development, was designed.
\end{abstract}

\section{Keywords}

Musical abilities, musical skills, screening, local criteria, integrated additive criteria, multicriteria optimization, mathematical model, decision-maker, "primary" quantitative assessment, weight coefficients, Pareto analysis, Churchman-Ackoff method

In opposite to the information explosion, driven by the rapid evolution of ICT, one of the problems faced by the current society is individual development.

We know that development is a multidimensional, systemic and integrated process. Or, it also could be related to the cultural formation of the society members. At the same time, it is known that a society with a high degree of general human culture is also a society with a high degree of economic, social and welfare development.

As a matter of fact that music is an important pillar of human existence through its ability to influence and induce different feelings and emotions, development and education of musical abilities with all its characteristic features can be considered as a real opportunity in the development of the individual with a high degree of general human culture, which meets the requirements of the moment. (stating that "When modes of music change, the fundamental laws of the State change with them", the great philosopher Plato in the "State" put the music in the relationship with the entire specific functioning mechanism of the fortress, and, in fact, emphasize that music can define a nation!).

In Pasat Natalia [1], the phenomenon was considered in terms of a much more specific aspect: "research into the phenomenon of musical abilities, analysis of the side effects and their degree of influence on the individual's development, as well as the development and presentation of a set of ICT services, enabling the testing and analysis of the musical abil- 
ities of the subjects, both individually and at the institution level”. It is obvious that the results obtained will have a particular impact on the multidimensional development of the individual, due to the fact that in the process of study and analysis there were involved experts with a rich experience and a high level of professional training.

According to the proposed approach, in Chapter 3, from Pasat Natalia [1], the study and analysis of the screening of the musical abilities and skills has been carried out with an impact on general human development, which does not exclude the possibility of reclassifying or supplementing the set of characteristics, starting from the following 3 local criteria:

1. The criterion based on the individual's profile (I);

2. The audiometric criterion (II);

3. Criterion of musical abilities (III).

Each of these is determined individually by different sets of characteristics (Table 1).

Table 1. Experimental model of the set of studied characteristics, divided according to the 3 local criteria, using Pareto method

\begin{tabular}{|c|c|c|c|c|c|c|c|}
\hline Nr. Crt. & $\begin{array}{l}\text { Nr. } \\
\text { Prt. }\end{array}$ & Particularities/Studied caracteristics & $\begin{array}{l}\text { Share } \\
\text { weight }\end{array}$ & $\begin{array}{c}\text { Share } \\
\text { weight } \\
(\%)\end{array}$ & $\begin{array}{l}\text { Aggegate } \\
\text { share } \\
\text { weight }\end{array}$ & $\begin{array}{c}\text { Pareto } \\
\text { AB }\end{array}$ & $\begin{array}{c}\text { Pareto } \\
\text { ABC }\end{array}$ \\
\hline CONST & 0.62 & & & & & \multirow{5}{*}{79.12} & \multirow{4}{*}{64.01} \\
\hline \multirow{7}{*}{$\mathbf{F 1}$} & 1 & $\begin{array}{l}\text { The ability to distinguish the rhythmic } \\
\text { formula }\end{array}$ & 0.3956 & 39.56 & 39.56 & & \\
\hline & 2 & The ability to distinguish sound frequencies & 0.2445 & 24.45 & 64.01 & & \\
\hline & 3 & The ability to distinguish sound duration & 0.1511 & 15.11 & 79.12 & & \\
\hline & 4 & Acoustic memory & 0.0934 & 9.34 & 88.46 & & \multirow{4}{*}{5.77} \\
\hline & 5 & $\begin{array}{c}\text { The ability to distinguish the musical } \\
\text { phrase development direction }\end{array}$ & 0.0577 & 5.77 & 94.23 & \multirow{3}{*}{20.88} & \\
\hline & 6 & $\begin{array}{l}\text { The ability to distinguish the musical } \\
\text { phrase mood / characteristics }\end{array}$ & 0.0357 & 3.57 & 97.80 & & \\
\hline & 7 & $\begin{array}{l}\text { The ability to distinguish the sound } \\
\text { intensity / loudness }\end{array}$ & 0.0220 & 2.20 & 100.00 & & \\
\hline \multirow{4}{*}{ F2 } & 1 & $\begin{array}{c}\text { The ability to distinguish the sound } \\
\text { loudness }\end{array}$ & 0.6180 & 61.80 & 61.80 & 61.80 & \\
\hline & 2 & $\begin{array}{l}\text { The ability to distinguish the sound } \\
\text { reference frequencies }\end{array}$ & 0.3820 & 38.20 & 100.00 & 38.20 & \\
\hline & 1 & Memory & 0.3903 & 39.03 & 39.03 & \multirow{3}{*}{78.06} & \multirow{2}{*}{63.15} \\
\hline & 2 & Concentration & 0.2412 & 24.12 & 63.15 & & \\
\hline \multirow{6}{*}{ F3 } & 3 & Physical co-ordination & 0.1491 & 14.91 & 78.06 & & \multirow{4}{*}{33.33} \\
\hline & 4 & Attention & 0.0921 & 9.21 & 87.27 & \multirow{5}{*}{21.93} & \\
\hline & 5 & Mathematical abilities & 0.0569 & 5.69 & 92.96 & & \\
\hline & 6 & Empathy & 0.0352 & 3.52 & 96.48 & & \\
\hline & 7 & Musical education degree & 0.0217 & 2.17 & 98.65 & & \multirow{2}{*}{3.52} \\
\hline & 8 & Age & 0.0134 & 1.34 & 99.99 & & \\
\hline
\end{tabular}

Which results in (Table 2):

Table 2. Three local criteria determined using Pareto method

\begin{tabular}{cccc}
\hline & & 3 CRITERIA CASE & \\
\hline F1 & CONST & 0.618034 \\
F2 & $\alpha$ & 0.5 \\
F3 & $\beta$ & & 0.309017 \\
& $\gamma$ & & 0.190983 \\
& & $\mathrm{~F}=\alpha * \mathrm{~F} 1+\beta * \mathrm{~F} 2+\gamma^{*} \mathrm{~F} 3$ & 1 \\
\hline
\end{tabular}


The following tools were used for the assessment of local criteria: testing, expert evaluation method, Pareto method. Moreover, the data gathered as a result of the proposed approach are collected and transmitted to the decision maker ("decision maker" or "analytical service"), which processes the data received, evaluates and classifies them by categories, after which they are passed to the work interface, which presents a report in graphical form and metrics for each of the local and integrated criteria used to evaluate the individual, subject to screening, according to the proposed approach.

\section{At the same time, the following challenges should be considered:}

1. Human development, studied from musical abilities point of view, is a multidimensional and complex process;

2. The proposed approach and the developed ICT tool are designed to be used both, individually and at the institution level;

3. 2 target groups are to be considered, potential beneficiaries of the proposed approach, at individual and institutional level, different assessment scales/metrics are needed;

4. There is a higher degree of risk for a decision to be taken if the scrutinizing individual does not cumulate the scale of the integrated scale but has "performing" skills in the development of other skills, being subject of research within the local criteria;

Taking into consideration the outlined above challenges, the following task may want to be formulated: based on the three local criteria/mentioned above (I), (II), (III)/, a model that facilitates the decision maker to evaluate the screening of individual's musical abilities and skills according to an integrated criterion, are going to be identified.

Remark 1: During the study and analysis phase in Pasat Natalia [1], it was found that the three local criteria have different share weights in the integrated value of the criterion. Thus, local criteria may be "hierarchically" organized (Table 4) as follows: musical skills criterion (I); audiometric criterion (II); the criterion of the individual's profile (III).

Remark 2: this model will succeed in determining a scale of values for the integrated criterion, which will allow the "primary" quantitative evaluation of the screening of the musical abilities and skills of the individual.

Considering those mentioned in the Remark 1 for the elaboration of the mathematical model for solving the above task, we will further note them as follows:

F - integrated criterion;

$\mathbf{F}_{\mathbf{1}}$ - musical abilities criterion (I);

$\mathbf{F}_{2}$ - audiometric criterion (II);

$\mathbf{F}_{3}$ - the criterion based on the individual's profile (III).

Then for $\mathbf{F}$ the following becomes valid:

$$
\boldsymbol{F}=\propto * \boldsymbol{F}_{\mathbf{1}}+\boldsymbol{\beta} * \boldsymbol{F}_{\mathbf{2}}+\boldsymbol{\gamma} * \boldsymbol{F}_{\mathbf{3}}
$$

In this formula, the coefficients $\boldsymbol{\alpha}, \boldsymbol{\beta}, \boldsymbol{\gamma}$ are the share weights mentioned in Remark 1. It is obvious, that in order to calculate the value of the integrated criterion, the values of the share - weighting factors $\boldsymbol{\alpha}, \boldsymbol{\beta}, \boldsymbol{\gamma}$ are to be determined. In other words, the mathematical model is actually a multicriteria optimization problem.

For multicriteria optimization methods, the initial ordering of the criteria is essential. Sometimes, the order is obvious, but there are cases when this is not a trivial problem and the solutions proposed by many experts are not relevant. One possibility would be to determine which expert's proposed order is "average", "typical" for a certain set of criteria. For this purpose, the Kemeni Median method [2] may be used for ordering.

The share-weighting factors $\boldsymbol{\alpha}, \boldsymbol{\beta}, \boldsymbol{\gamma}$ are supposed to reflect the quality importance of the specific, relevant local criteria. These values are often selected based on the criteria for the task studied and the opportunities for their implementation. $\boldsymbol{\alpha}$, $\boldsymbol{\beta}, \boldsymbol{\gamma}$ values determine the importance of the reference optimality criterion and determine the quantitative measurement of a preferential criterion against other optimality criteria. An important point to take in account is that these coefficients have to satisfy the condition:

$$
\propto+\boldsymbol{\beta}+\boldsymbol{\gamma}=1(\text { or } \propto+\boldsymbol{\beta}+\boldsymbol{\gamma}=100)
$$

In light of this, the following the question may be formulated: "How to choose the numerical values of share-weighting coefficients $\boldsymbol{\alpha}, \boldsymbol{\beta}, \boldsymbol{\gamma}$ ?”. We can get an answer for this question if we have some additional information on the importance of local optimization criteria in the formulated task.

In the specialized literature there are several methods for calculating the share-weighting factors $\boldsymbol{\alpha}, \boldsymbol{\beta}, \boldsymbol{\gamma}$ for the local criteria in the integrated criterion:

1. Methods for criteria pair comparison and derivatives of these methods;

2. Methods that use indicators interrelation analytical dependencies, which determine the criteria importance;

3. Formal methods, such as the method of successive comparison of criteria, known as the Churchman-Ackoff method. [3];

In the following, we will exemplify the Churchman-Ackoff method. According to this method:

- Each criterion $\mathbf{F}_{\mathbf{i}}$, (i = 1,2,3 ... n, where n-number of criteria) is given a certain share-weight, a real non-negative 
number $\mathbf{p}_{\mathbf{i}}$;

- If the $\mathbf{F}_{\mathbf{i}}$ criterion is preferable to the criterion $\mathbf{F}_{\mathbf{j}}(\mathrm{j}=1,2,3 \ldots \mathrm{n}, \mathbf{j} \neq \mathrm{i})$, then $\mathbf{p}_{\mathbf{i}}>\mathbf{p}_{\mathbf{j}}$, and if these criteria are equivalent, then $\mathbf{p}_{\mathbf{i}}=\mathbf{p}_{\mathbf{j}}$;

- If $\mathbf{p}_{\mathbf{i}}$ and $\mathbf{p}_{\mathbf{j}}$ are estimates of $\mathbf{F}_{\mathbf{i}}$ and $\mathbf{F}_{\mathbf{j}}$, then the estimate $\left(\mathbf{p}_{\mathbf{i}}+\mathbf{p}_{\mathbf{j}}\right)$ corresponds to the common influence of these criteria.

Criteria are classified according to preference, according to Cherchmen-Akoff's method (Table 4). At the same time, we agree to assume that the $\mathbf{F}_{1}$ criterion is the most important, followed by $\mathbf{F}_{2}$, and so on. until $\mathbf{F}_{\mathbf{n}}$.

The decision factor defines the preliminary numerical estimates $\mathbf{p}_{\mathbf{i}}$ for each $\mathbf{F}_{\mathbf{i}}$ criterion. Typically, the most important criterion is defined as a share-weight of 100 points, the remaining criteria are rated between 0 and 100 depending on their importance. Then the decision factor compares the $\mathbf{F}_{\mathbf{1}}$ criterion estimates with the sum of the other criteria from $\mathbf{F}_{2}$ to $\mathbf{F}_{\mathbf{n}}$. (if we have $\mathbf{n}$ criteria).

If the $\mathbf{F}_{\mathbf{1}}$ criterion is more important than the others, the decision maker corrects the estimates so that the following relationship takes place.

Otherwise, the following condition is required:

$$
p_{i}>\sum_{i=2}^{i=n} p_{i}
$$

$$
\boldsymbol{p}_{\boldsymbol{i}}<\sum_{i=2}^{i=n} \boldsymbol{p}_{\boldsymbol{i}}
$$

If the $\mathbf{F}_{\mathbf{1}}$ criterion is less important than the sum of all other criteria, then for estimates clarification, its estimate is compared by preference to the sum of estimates of other criteria. After the evaluation of the $\mathbf{F}_{\mathbf{1}}$ criterion becomes more important than the sum of the estimates of the criteria $\mathbf{F}_{2} \ldots \mathbf{F}_{\mathbf{k}} \ldots \mathbf{F}_{\mathbf{n}}(\mathrm{k}>=2)$, this criterion is excluded from the consideration and instead of the evaluation of the $\mathbf{F}_{\mathbf{1}}$ criterion, the criterion $\mathbf{F}_{\mathbf{2}}$ is referenced and the estimates are corrected. The process continues until the estimates of all the criteria are corrected.

If the criteria number is considerable, it is recommended that they are broken down into criteria groups by importance, so that one of the criteria that is most important, to adjust the estimates of all criteria is to include them in all groups. This will make it possible to simplify the process of assessing the criteria.

To calculate the share-weighting coefficients for the $\mathbf{F}_{\mathbf{i}}$ criteria, we will use the following formula:

$$
\propto_{i}=\frac{p_{i}}{\sum_{i=1}^{i=n} p_{i}}, \quad \mathrm{i}=1,2, \ldots, \mathrm{n}
$$

For our case, $n=3, i=1,2,3$, where $\propto=\propto_{1}, \beta=\propto_{2}$ and $\gamma=\propto_{3}$.

According to the first experimental data, the following results were obtained for each set of characteristics (presented in Table 1, Table 2), belonging to each of the criteria (presented in Table 3, Table 4, Table 5), recalculated according to the purpose of the evaluation process.

Let us to consider the musical hearing characteristics for the mentioned criteria, that are much more important in the screening of musical abilities and skills. Following, we define for this case the 3 goals below, that highlight these capabilities of the subject exposed to the testing process (Table 3).

Table 3. Goals prioritisation according to the decision-maker

\begin{tabular}{ccc}
\hline Nr. & Goal & Goal Notation \\
\hline 1 & Musical hearing testing & A \\
2 & Musical hearing performance based selection & B \\
3 & Hearing characteristics monitoring & C \\
\hline
\end{tabular}

Table 4. Ranking assignement of the three criteria for the Churchman-Ackoff method

\begin{tabular}{ccccc}
\hline Nr. & Criterion $\left(\mathbf{C}_{\mathbf{i}}, \mathbf{i}=\mathbf{1}, \mathbf{2}, \mathbf{3}\right)$ & Criterion notation & Weight notation & Rank-Value \\
\hline 1 & Musical hearing characteristics & F1 & A & 3 \\
2 & Audiometry & F2 & B & 2 \\
3 & General profile & F3 & $\Gamma$ & 1
\end{tabular}


Table 5. The share-weight table of the listed goals according to criteria determined above

\begin{tabular}{lllll}
\hline Criterion & Goal & A & B & C \\
\hline & & & 0.9 & 0.8 \\
& $\mathrm{C}_{1}$ & 0.9 & 0.5 & 0.5 \\
& $\mathrm{C}_{2}$ & 0.7 & 0.5 & 0.6 \\
& $\mathrm{C}_{3}$ & 0.2 & 0.7 & 0.666667 \\
\hline
\end{tabular}

Table 6. Results recalculated for the particularities of local criterion F1, according to the Churchman-Ackoff method, depending on the purpose

\begin{tabular}{ccc}
\hline Nr. & Particularities/studied caracteristics & Normalized coefficients \\
\hline $\mathbf{7}$ & The ability to distinguish the rhythmic formula & 0.25 \\
$\mathbf{6}$ & The ability to distinguish sound frequencies & 0.214285714 \\
$\mathbf{5}$ & The ability to distinguish sound duration & 0.178571429 \\
$\mathbf{4}$ & Acoustic memory & 0.142857143 \\
$\mathbf{3}$ & The ability to distinguish the musical phrase development direction & 0.107142857 \\
$\mathbf{2}$ & The ability to distinguish the musical phrase mood/characteristics & 0.071428571 \\
$\mathbf{1}$ & The ability to distinguish the sound intensity/loudness & 0.035714286 \\
\hline
\end{tabular}

Table 7. The share - weight table for the particularities of local criterion F1

\begin{tabular}{|c|c|c|c|c|}
\hline Particularities/studied caracteristics & Goal & A & B & C \\
\hline 7 & & 0.9 & 0.9 & 0.9 \\
\hline 6 & & 0.9 & 0.9 & 0.9 \\
\hline 5 & & 0.9 & 0.5 & 0.5 \\
\hline 4 & & 0.6 & 0.7 & 0.7 \\
\hline 3 & & 0.2 & 0.7 & 0.4 \\
\hline 2 & & 0.4 & 0.7 & 0.3 \\
\hline 1 & & 0.5 & 0.5 & 0.5 \\
\hline & & 0.732143 & 0.75 & 0.689286 \\
\hline
\end{tabular}

Table 8. Results recalculated for the particuliarities of local criterion F2, according to the Churchman-Ackoff method, depending on the purpose

\begin{tabular}{crc}
\hline Nr. & Particularities/studied caracteristics & Normalized coefficients \\
\hline $\mathbf{2}$ & The ability to distinguish the sound loudness & 0.67 \\
$\mathbf{1}$ & The ability to distinguish the sound reference frequencies & 0.33 \\
\hline
\end{tabular}

Table 9. The share-weight table of the studied characteristics for local criterion F2

\begin{tabular}{|c|c|c|c|c|}
\hline Particularities/studied caracteristics & Goal & A & B & C \\
\hline 2 & & 0.8 & 0.4 & 0.5 \\
\hline 1 & & 0.6 & 1 & 0.5 \\
\hline & & 0.733333 & 0.6 & 0.5 \\
\hline
\end{tabular}


Table 10. Results recalculated for the particuliarities of local criterion F3, according to the Churchman-Ackoff method, depending on the purpose

\begin{tabular}{ccc}
\hline Nr. & Particularities/studied caracteristics & Normalized coefficients \\
\hline $\mathbf{8}$ & Memory & 0.22 \\
$\mathbf{7}$ & Concentration & 0.19 \\
$\mathbf{6}$ & Physical co-ordination & 0.17 \\
$\mathbf{5}$ & Attention & 0.14 \\
$\mathbf{4}$ & Mathematical abilities & 0.11 \\
$\mathbf{3}$ & Empathy & 0.08 \\
$\mathbf{2}$ & Musical education degree & 0.06 \\
$\mathbf{1}$ & Age & 0.03 \\
\hline
\end{tabular}

Table 11. The share-weight table of the listed particularities for local criterion F3 (Table 10)

\begin{tabular}{|c|c|c|c|}
\hline $\begin{array}{c}\text { Goal } \\
\text { Particularities/studied caracteristics }\end{array}$ & A & B & C \\
\hline 8 & 0.6 & 0.7 & 0.7 \\
\hline 7 & 0.2 & 0.4 & 0.7 \\
\hline 6 & 0.2 & 0.2 & 0.2 \\
\hline 5 & 0.4 & 0.7 & 0.6 \\
\hline 4 & 0.3 & 0.3 & 0.5 \\
\hline 3 & 0.2 & 0.5 & 0.5 \\
\hline 2 & 0.8 & 0.8 & 0.6 \\
\hline 1 & 0.5 & 0.8 & 0.2 \\
\hline & 0.369444 & 0.511111 & 0.544444 \\
\hline
\end{tabular}

According to the calculations presented in Table 6, Table 7, Table 8, Table 9, Table 10, and Table 11, the final recalculated weightings for our case, screening of musical abilities and skills are shown in Table 12.

Table 12. The tendency to achieve the proposed goals according to the Churchman-Ackoff method

\begin{tabular}{lcccc}
\hline & Goal & A & B & C \\
Weight for Criteria & & 1.834921 & 1.86111 & 1.73373
\end{tabular}

Or, for $\mathbf{F}$ - integrated criterion for screening of musical abilities and skills, which consist from:

$\mathbf{F}_{1}$ - musical abilities criterion;

$\mathbf{F}_{2}$ - audiometric criterion;

$\mathbf{F}_{3^{-}}$the criterion based on the individual's profile.

based on the 3 mentioned above goals (Table 3), that highlight the special capabilities of the subject exposed to the testing process prezented by A, B and C, could be presented by the next model:

$$
F=1,834921 * F_{1}+1,861111 * F_{2}+1,73373 * F_{3}
$$

Having this formula, it is possible to apply screening of the musical abilities and skills for different test subjects starting from the most different assumptions and creating in such way a data base for decision makers in the mentioned area.

In conclusion:

- The base purpose of this study and analysis is to put forward the "primary" mathematical model for quantitative evaluation of the musical abilities and skills screening;

- $\quad$ Based on the results obtained in Pasat Natalia [1], a mathematical model has been identified that facilitates the decision maker to evaluate the integrated criterion in the screening of musical abilities and skills with an impact on general human development. 
- It is clear that not all the challenges mentioned above in the model can be taken into account by the proposed approach, due to the complexity of the distinction of the particularities considered and, finally, the general criteria. The original mathematical model that was built could change the input and output data sets, based on the new features and objectives, their classification or the target audience. It also remains an open question the parameters of the ideal screening and the range of results for the test subjects. So far, this has not been the purpose of this paper.

- It remain open the objectives and purpose of increasing the accuracy of screening results, as well as to extend the applicability of the proposed mathematical model, not only for quantitative assessment and screening of musical skills and abilities, but also for the consideration of a wide range of other subjects of activity and fields of research.

\section{References}

[1] Pasat Natalia. (2018). ICT Services for screening of musical abilities and skills, bachelor thesis, Chişinău, 2018, TUM, FCIM, DIIS, Applied Informatics.

[2] Kemeny, J. and Snell, L. (1962). Mathematical Models in the Social Sciences. Ginn, Boston.

[3] Churchman and Ackoff. (1954). The method of successive comparisons. 